\title{
CHARM PRODUCTION, MIXING AND CP VIOLATION*
}

\author{
TADEusz Lesiak \\ on behalf of the LHCb Collaboration \\ The Henryk Niewodniczański Institute of Nuclear Physics PAN \\ Radzikowskiego 152, 31-342 Kraków, Poland \\ and \\ Tadeusz Kościuszko Cracow University of Technology \\ Faculty of Physics, Mathematics and Computer Science \\ Podchorążych 1, 30-084 Kraków, Poland \\ (Received April 14, 2014) \\ Recent results on mixing, $\mathrm{CP}$ violation and spectroscopy in the charm \\ sector from the LHCb experiment are briefly reviewed. \\ DOI:10.5506/APhysPolB.45.1469 \\ PACS numbers: 14.65.Dw, 14.40.Lb, 14.20.Lq, 13.25.Ft
}

\section{Introduction}

The phenomenon of quantum mechanical mixing arises between neutral meson particle and anti-particle flavor eigenstates, like $K^{0}-\bar{K}^{0}, B_{d}^{0}-\bar{B}_{d}^{0}$, $B_{s}^{0}-\bar{B}_{s}^{0}$ and $D^{0}-\bar{D}^{0}$. It is quantitatively characterized by two dimensionless parameters

$$
x=\frac{\Delta m}{\Gamma}, \quad y=\frac{\Delta \Gamma}{\Gamma},
$$

where $\Delta m=m_{2}-m_{1}\left(\Delta \Gamma=\Gamma_{2}-\Gamma_{1}\right)$ is the mass (width) difference between the mass eigenstates, and $\Gamma=\left(\Gamma_{1}+\Gamma_{2}\right) / 2$ is the average width. The mixing is an important source of information about electroweak interactions [1], in particular about the Cabibbo-Kobayashi-Maskawa matrix [2]. It is also sensitive to the exchange of virtual particles which are not described by the Standard Model (SM). The latter would constitute New Physics (NP).

\footnotetext{
* Presented at the Cracow Epiphany Conference on the Physics at the LHC, Krakow,
} Poland, January 8-10, 2014. 
Three kinds of CP violation (CPV) can be distinguished in meson decays:

1. CP violation in decay, commonly known as direct CPV,

2. CP violation in mixing,

3. CP violation in the interference between a direct decay, $D^{0} \rightarrow f$, and a process with the mixing in the first stage, $\bar{D}^{0} \rightarrow D^{0} \rightarrow f$, where $f$ is a final state.

The last two categories are also labeled together as indirect CPV.

The LHCb detector [3] is a single-arm forward spectrometer covering the pseudorapidity range $2<\eta<5$. The apparatus is designed for the study of $b$ quarks, but it also offers very favorable conditions for measurements in the charm sector. The cross-section for the production of $c \bar{c}$ pairs was measured to be of $1419 \pm 134 \mu \mathrm{b}$ [4], i.e. about 20 times bigger than the production of $b \bar{b}$ pairs [5]. The LHCb experiment is also equipped with a flexible and efficient trigger system, suited in particular for reconstruction of charm hadrons [6].

The data used in the studies described below comprise $1.1 \mathrm{fb}^{-1}\left(0.65 \mathrm{fb}^{-1}\right.$ in the case of the search for the $\Xi_{c c}^{+}$baryon) collected in $p p$ collisions at $\sqrt{s}=7 \mathrm{TeV}$ in 2011. The three subsequent sections will be devoted to the LHCb results concerning charm mixing, searches for CPV violation and spectroscopy studies in the charm sector.

\section{Observation of charm mixing}

Mixing is now well established in the strange and beauty sectors where it is described by SM box-diagrams containing up-type quarks. The $D^{0}-\bar{D}^{0}$ oscillations, occurring via loops involving down-type quarks, are predicted to be very small, with the values of $x$ and $y$ less than or of the order of $1 \%$ level [7]. Moreover, the precision of these theoretical expectations is limited due to the presence of substantial, non-perturbative, long-distance contributions. In view of the above, it is no wonder that the first evidence for charm mixing was reported only in 2007, first in $e^{+} e^{-}$annihilation at the $B$-factories $[8,9]$, and then also in $p \bar{p}$ collisions at the Tevatron [10]. Soon the no-mixing hypothesis was excluded with a significance substantially exceeding $5 \sigma$ [11]. This was obtained, however, by combining results of different measurements. In 2012 , the $\mathrm{LHCb}$ experiment provided the first incontrovertible observation of charm mixing [12] excluding the no-mixing hypothesis with a probability corresponding to $9.1 \sigma$. This observation was recently confirmed by the CDF [13] and Belle [14] experiments. 
The LHCb measurement [12] is based on the selection of prompt $D^{*+} \rightarrow$ $D^{0} \pi_{\mathrm{s}}^{+}$decays that are consistent with production at the primary vertex. The charge of the soft pion $\pi_{\mathrm{s}}$ provides the tag of the $D$ meson's flavor at production. The decay chain $D^{*+} \rightarrow D^{0}\left(\rightarrow K^{-} \pi^{+}\right) \pi_{\mathrm{s}}^{+}$, designated as rightsign (RS), is dominated by a Cabibbo-favored (CF) decay. The process $D^{*+} \rightarrow D^{0}\left(\rightarrow K^{+} \pi^{-}\right) \pi_{\mathrm{s}}^{+}$, labeled as wrong-sign (WS), encompasses contributions from the doubly-Cabibbo-suppressed (DCS) process $D^{0} \rightarrow K^{+} \pi^{-}$ and from a $D^{0}-\bar{D}^{0}$ mixing followed by the $\mathrm{CF}$ decay $\bar{D}^{0} \rightarrow K^{+} \pi^{-}$. The time-dependent ratio, $R$, of WS to RS decay rates is approximated by

$$
R(t) \approx R_{D}+\sqrt{R_{D}} y^{\prime} \frac{t}{\tau}+\frac{x^{\prime 2}+y^{\prime 2}}{4}\left(\frac{t}{\tau}\right)^{2}
$$

where $R_{D}$ denotes the ratio of DCS and CF decay rates, $t / \tau$ is the decay time expressed in units of the average $D^{0}$ lifetime, $x^{\prime}=x \cos \delta+y \sin \delta$, $y^{\prime}=-x \sin \delta+y \cos \delta$ and $\delta$ is the strong phase between the DCS and CF amplitudes. Substantial samples of $8.4 \times 10^{6} \mathrm{RS}$ and $3.6 \times 10^{4} \mathrm{WS}$ decays have been reconstructed. The WS/RS ratio was determined in thirteen $D^{0}$ decay bins, chosen to have a similar population of candidates (Fig. 1). The mixing parameters were determined in a binned $\chi^{2}$ fit to the parametrization according to Eq. (2). The values of the mixing parameters were determined as $x^{\prime 2}=(-0.9 \pm 1.3) \times 10^{-4}$ and $y^{\prime}=(7.2 \pm 2.4) \times 10^{-3}$, where the uncertainties include statistical and systematic sources.

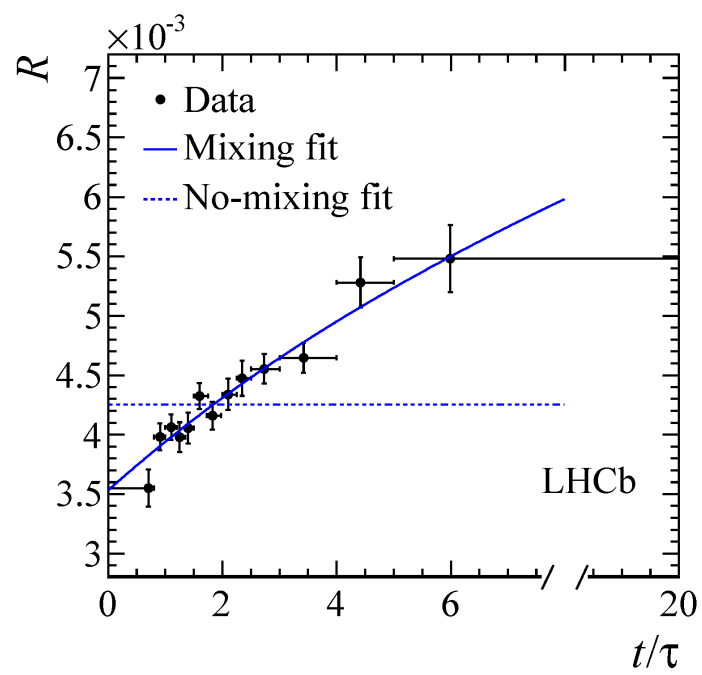

Fig. 1. The ratio $R$ of wrong-sign $D^{0} \rightarrow K^{+} \pi^{-}$to right-sign $D^{0} \rightarrow K^{-} \pi^{+}$yields (points) versus the decay time. The solid (dashed) line corresponds to mixing allowed (no-mixing) fits, respectively. 
This approach was recently extended by the $\mathrm{LHCb}$ Collaboration in search for CPV using a data sample of $3 \mathrm{fb}^{-1}$ and studying $D^{0}$ and $\overline{D^{0}}$ decays separately [15].

\section{Searches for CP violation in the charm sector}

In the Standard Model, the CPV amplitudes are due to Cabibbo-suppressed decays and are expected to be very small, i.e. at most at the few permille level. They can, however, be significantly enhanced by the presence of NP. The LHCb experiment has an extensive programme of searches for CPV phenomena in the charm sector. It encompasses two-, three- and fourbody decays of both neutral and charged $D$ mesons.

\subsection{Search for $C P$ violation in $D^{0} \rightarrow K^{+} K^{-}$and $D^{0} \rightarrow \pi^{+} \pi^{-}$decays}

The search for time-integrated, direct $\mathrm{CP}$ violation in the decays to $\mathrm{CP}$ eigenstates $D^{0} \rightarrow K^{+} K^{-}$and $D^{0} \rightarrow \pi^{+} \pi^{-}$was performed by the $\mathrm{LHCb}$ Collaboration using two tags of the $D^{0}$ flavor. The first approach $\left(\pi_{\mathrm{s}}\right.$-tag $)$ [16] used the charge of the soft pion from a $D^{*+}$ decay, whereas in the second one ( $\mu$-tag) [17] the $D^{0}$ flavor is determined from the charge of the muon originating from the decay $b$-hadron $\rightarrow D^{0} \mu^{-} X$. Here, the $X$ denotes any other particle(s) produced in the semileptonic $b$-hadron decay, which are not reconstructed. The measured (raw) asymmetry for a CP eigenstate is defined as

$$
A_{\text {raw }}(f)=\frac{N\left(D^{0} \rightarrow f\right)-N\left(\bar{D}^{0} \rightarrow f\right)}{N\left(D^{0} \rightarrow f\right)+N\left(\bar{D}^{0} \rightarrow f\right)},
$$

where $f=K^{+} K^{-}, \pi^{+} \pi^{-}$and $N$ corresponds to the observed yield for the given decay. The raw asymmetries can be reliably approximated as a sum of components that are due to $\mathrm{CP}$ violation and to detector effects. The latter cancel in the difference between the raw asymmetries for the two final states in question

$$
\Delta A_{\mathrm{CP}}=A_{\mathrm{CP}}\left(K^{+} K^{-}\right)-A_{\mathrm{CP}}\left(\pi^{+} \pi^{-}\right)=A_{\text {raw }}\left(K^{+} K^{-}\right)-A_{\text {raw }}\left(\pi^{+} \pi^{-}\right) .
$$

The total number of signal candidates reconstructed for the $\pi_{\mathrm{s}}$-tag was $2.24 \times$ $10^{6}$ for $D^{0} \rightarrow K^{+} K^{-}$decays and $0.69 \times 10^{6}$ for $D^{0} \rightarrow \pi^{+} \pi^{-}$. The respective numbers of events for the $\mu$-tag were $5.6 \times 10^{5}$ and $2.2 \times 10^{5}$. The difference in $\mathrm{CP}$ asymmetry between the $D^{0} \rightarrow K^{+} K^{-}$and in $D^{0} \rightarrow \pi^{+} \pi^{-}$decays was measured to be consistent with null: $\Delta A_{\mathrm{CP}}=(-0.34 \pm 0.15 \pm 0.10) \%$ for the $\pi_{\mathrm{s}}$-tag and $\Delta A_{\mathrm{CP}}=(+0.49 \pm 0.30 \pm 0.14) \%$ for the $\mu$-tag. This yields an average of $\Delta A_{\mathrm{CP}}=(-0.15 \pm 0.16) \%$. Thus, no evidence for direct $\mathrm{CP}$ violation in the charm sector is found. 


\subsection{Measurement of indirect $C P$ asymmetries $A_{\Gamma}$}

The CPV can also show up as the asymmetry of the inverse of effective lifetimes $(\hat{\Gamma})$ of $D^{0}$ and $\bar{D}^{0}$ mesons decaying to the same CP eigenstate $f=K^{+} K^{-}$or $\pi^{+} \pi^{-}$. The observable $A_{\Gamma}$ is defined as

$$
A_{\Gamma}=\frac{\hat{\Gamma}\left(D^{0} \rightarrow f\right)-\hat{\Gamma}\left(\bar{D}^{0} \rightarrow f\right)}{\hat{\Gamma}\left(D^{0} \rightarrow f\right)+\hat{\Gamma}\left(\bar{D}^{0} \rightarrow f\right)} \approx \frac{A_{\mathrm{m}}+A_{\mathrm{d}}}{2} y \cos \phi-x \sin \phi
$$

where $A_{\mathrm{m}}$ and $A_{\mathrm{d}}$ are the asymmetries due to mixing and decay, respectively, and $\phi$ is the CP-violating relative phase between mixing and decay. The asymmetry (5) is considered as a measure of indirect CPV, as the effects of direct CPV are constrained to be small [11] compared to the present experimental precision [18].

The LHCb Collaboration extracted the mean lifetimes of the $D^{0}$ and $\bar{D}^{0}$ separately via a fit to their decay times [19] (Fig. 2). The flavor of the $D$ meson was tagged by the charge of the soft pion from the decay $D^{*+} \rightarrow D^{0} \pi^{+}$. The $A_{\Gamma}$ asymmetries were measured to be of $A_{\Gamma}\left(K^{+} K^{-}\right)=$ $(0.35 \pm 0.62 \pm 0.12) \times 10^{-3}$ and $A_{\Gamma}\left(\pi^{+} \pi^{-}\right)=(0.33 \pm 1.06 \pm 0.14) \times 10^{-3}$ and indicate the absence of indirect $\mathrm{CPV}$ at the current level of accuracy. These results are the world's best measurements of these quantities.

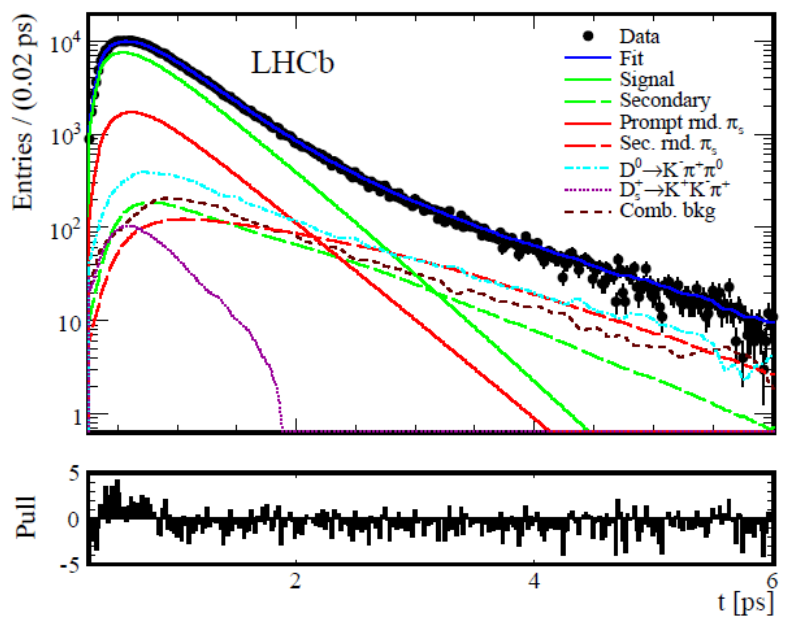

Fig. 2. An example fit to one decay time distribution used in the $A_{\Gamma}$ measurement. The data are $\bar{D}^{0} \rightarrow K^{+} K^{-}$candidates from a sample of data taken with magnet polarity down. 


\subsection{Search for direct $C P$ violation in $D^{+} \rightarrow \phi \pi^{+}$and $D_{s}^{+} \rightarrow K_{\mathrm{S}}^{0} \pi^{+}$decays}

The LHCb Collaboration has performed a search for direct $\mathrm{CP}$ violation in charged $D$ meson decays [20]. The process $D^{+} \rightarrow \phi\left(\rightarrow K^{+} K^{-}\right) \pi^{+}$has been studied with the decay $D^{+} \rightarrow K_{\mathrm{S}}^{0} \pi^{+}$used as a control channel. For the latter, the $\mathrm{CP}$ violating effects are expected to be negligible as it is dominated by the tree diagram amplitudes. As part of the same analysis, a measurement of a second CP asymmetry was made with the decay $D_{s}^{+} \rightarrow K_{\mathrm{S}}^{0} \pi^{+}$. In the latter case, the $D_{s}^{+} \rightarrow \phi \pi^{+}$decay was used as a control channel. The yields of signal events were $1.58 \times 10^{6}$ for the decay $D^{+} \rightarrow \phi \pi^{+}$and $2.6 \times 10^{4}$ for $D_{s}^{+} \rightarrow K_{\mathrm{S}}^{0} \pi^{+}$. The raw asymmetries were defined as

$$
A_{\text {raw }}=\frac{N\left(D_{(s)}^{+} \rightarrow f\right)-N\left(D_{(s)}^{-} \rightarrow \bar{f}\right)}{N\left(D_{(s)}^{+} \rightarrow f\right)-N\left(D_{(s)}^{-} \rightarrow \bar{f}\right)} .
$$

The CP asymmetry in $D^{+} \rightarrow \phi \pi^{+}$is the difference between the raw asymmetries for the signal and control channels with a correction of $(-0.028 \pm$ 0.028)\% due to the CPV in the neutral kaon system

$$
A_{\mathrm{CP}}\left(D^{+} \rightarrow \phi \pi^{+}\right)=A_{\text {raw }}\left(\phi \pi^{+}\right)-A_{\text {raw }}\left(K_{\mathrm{S}}^{0} \pi^{+}\right)+A_{\mathrm{CP}}\left(K^{0} / \bar{K}^{0}\right) .
$$

The symmetric expression was applied for the measurement of the $A_{\mathrm{CP}}\left(D_{s}^{+}\right.$ $\left.\rightarrow K_{\mathrm{S}}^{0} \pi^{+}\right)$. The results of this study were $A_{\mathrm{CP}}\left(D^{+} \rightarrow \phi \pi^{+}\right)=(-0.04 \pm$ $0.14 \pm 0.14) \%$ and $A_{\mathrm{CP}}\left(D_{s}^{+} \rightarrow K_{\mathrm{S}}^{0} \pi^{+}\right)=(0.61 \pm 0.83 \pm 0.14) \%$, and are fully consistent with the lack of direct CPV in the charm system.

\subsection{Search for CP violation in three- and four-body decays of $D$ mesons}

Multibody decays of charm mesons provide rich resonance structures with interfering amplitudes and, moreover, are sensitive to CPV localized in certain phase space regions. The LHCb Collaboration has performed searches for such local CP asymmetries in several multibody $D$ decay modes. Two of them are briefly discussed below.

A search for CP asymmetries in four-body, singly-Cabibbo-suppressed $D^{0}$ decays was carried out at $\mathrm{LHCb}$ with samples of of $5.7 \times 10^{4} D^{0} \rightarrow$ $K^{+} K^{-} \pi^{+} \pi^{-}$and $3.3 \times 10^{5} D^{0} \rightarrow \pi^{+} \pi^{-} \pi^{+} \pi^{-}$decays from the chain $D^{*+} \rightarrow$ $D^{0} \pi_{\mathrm{s}}^{+}$[21]. As in most abovementioned studies, the charge of the soft pion identified the flavor of the $D$ meson at production. In this case, the phase space can be described in terms of five invariant mass-squared combinations: $s(1,2), s(2,3), s(3,4), s(1,2,3)$ and $s(2,3,4)$ (with the notation: $D^{0} \rightarrow$ 1253 ). This phase space was partitioned into $N_{\text {bin }}$ independent volumes 
to give a similar number of events in each $5 \mathrm{D}$ hypercube. The variable $S_{\mathrm{CP}}^{i}$ measured the significance of CPV in the volume $i$

$$
S_{\mathrm{CP}}^{i}=\frac{N_{i}\left(D^{0}\right)-\alpha N_{i}\left(\bar{D}^{0}\right)}{\sqrt{\alpha\left[\sigma_{i}^{2}\left(D^{0}\right)+\sigma_{i}^{2}\left(\bar{D}^{0}\right)\right]}}, \quad \alpha=\frac{\sum_{i} N_{i}\left(D^{0}\right)}{\sum_{i} N_{i}\left(\bar{D}^{0}\right)} .
$$

Here, the symbol $N_{i}$ denotes the number of the decays and $\sigma_{i}$ is the associated uncertainty. The normalization constant $\alpha$ was used to remove any global production or detection asymmetries.

In the absence of any CPV, it is expected that the $S_{\mathrm{CP}}$ is Gaussiandistributed with a mean of zero and with a width of one. The presence of $\mathrm{CPV}$ can be identified by the following $\chi^{2}$ statistic

$$
\chi^{2}=\sum_{i}\left(S_{\mathrm{CP}}^{i}\right)^{2}
$$

with $\left(N_{\text {bin }}-1\right)$ degrees of freedom from which a $p$-value is determined. The latter gives the probability of getting the observed results if the no CPV hypothesis is assumed. Figure 3 shows the $S_{\mathrm{CP}}^{i}$ distributions for both decays in questions. Neither of them shows evidence of local CPV. The $p$-values were estimated as $9.1 \%$ for $D^{0} \rightarrow K^{+} K^{-} \pi^{+} \pi^{-}$and $41 \%$ for $D^{0} \rightarrow$ $\pi^{+} \pi^{-} \pi^{+} \pi^{-}$(partitioned with 32 and 128 bins, respectively).
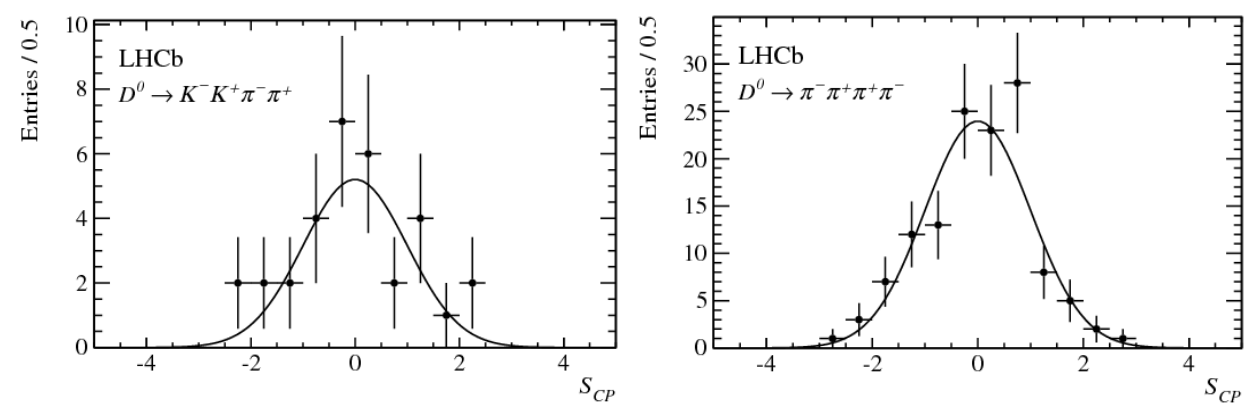

Fig. 3. Distributions of $S_{\mathrm{CP}}$ for $D^{0} \rightarrow K^{+} K^{-} \pi^{+} \pi^{-}$decays partitioned with 32 bins (left) and for $D^{0} \rightarrow \pi^{+} \pi^{-} \pi^{+} \pi^{-}$decays partitioned with 128 bins (right). The points are the data distribution and the solid line shows a reference Gaussian distribution corresponding to the no CPV hypothesis.

The LHCb has also performed a similar study on the sample of $2.7 \times 10^{6}$ three-body decays $D^{+} \rightarrow \pi^{-} \pi^{+} \pi^{+}$[22]. In this case, the decay dynamics were described by two invariants $s_{\text {low }}$ and $s_{\text {high }} i . e$. the lowest and highest invariant mass squared combination $M^{2}\left(\pi^{+} \pi^{-}\right)$. No evidence for localized CPV has been found and the $p$-values span the range (50-99)\% for various numbers of partitions. 


\section{Charm spectroscopy}

\subsection{Quantum numbers of the $X(3872)$}

The narrow state $X(3872)$ was discovered by the Belle experiment [23] in 2003 in $B$ meson decays but still lacks a clear-cut interpretation. The $C$ parity of the $X$ was determined to be positive since the radiative decay $X(3872) \rightarrow J / \psi \gamma$ has been observed [24, 25]. However, extensive studies performed by BaBar, Belle and CDF were not able to fully remove the controversy about the spin-parity $\left(J^{\mathrm{P}}\right)$ of the $X$. The two remaining hypotheses are $1^{+}$and $2^{-}$. The former assignment would lead to the interpretation of $X$ as either a $D^{* 0} \bar{D}^{0}$ molecule [26], a tetraquark [27] or a charmonium state $\chi_{c 1}\left(2^{3} P_{1}\right)$. The case of $2^{-}$would point toward a charmonium $\eta_{c 2}\left(1^{1} D_{2}\right)$. In order to distinguish between the above two hypotheses, a full analysis of angular correlations in an exclusive production environment, such as a $B$ meson decay, is necessary.

The LHCb Collaboration has studied the complete five-dimensional angular distribution of the $B^{+} \rightarrow X(3872) K^{+}, X(3872) \rightarrow J / \psi \pi^{+} \pi^{-}, J / \psi \rightarrow$ $\mu^{+} \mu^{-}$decay chain [28]. The data sample comprised $313 \pm 26$ signal candidates. The likelihood-ratio test with the test statistic $t=-2 \ln \left[\mathcal{L}\left(2^{-}\right) / \mathcal{L}\left(1^{+}\right)\right]$ was applied to discriminate between the $1^{+}$and $2^{-}$assignments. The value observed in the data, $t_{\text {data }}=99$, clearly favors the $1^{+}$hypothesis. The $2^{-}$ assignment was ruled out with a significance of $8.4 \sigma$. Thus the $J^{\mathrm{PC}}$ quantum numbers of the $X(3872)$ were unambiguously determined by the LHCb to be $1^{++}$.

\subsection{Spectroscopy of $D_{J}$ mesons}

Many charm meson states that are predicted in the quark model [29] have not yet been observed experimentally. Thus, more information about the properties and especially quantum numbers of excited $D$ mesons is needed. The states with $P=(-1)^{J}$ are called natural parity states while those with $P=(-1)^{J+1}$ are labeled as unnatural parity ones.

A search for excited charmed mesons, labeled as $D_{J}$, was performed by the LHCb Collaboration [30] using the inclusive reactions $p p \rightarrow D^{+} \pi^{-} X$, $p p \rightarrow D^{0} \pi^{+} X$ and $p p \rightarrow D^{*+} \pi^{-} X$, where $X$ denotes a system composed of any number of charged and neutral particles. The states $D_{1}(2420)$ and $D_{2}^{*}(2460)$ have been clearly seen, together with several structures observed in the mass region between 2500 and $3000 \mathrm{MeV} / c^{2}$ (Table I). For the latter, the yields of observed decays span the range $(7-675) \times 10^{3}$ events, while the respective significances are between 6.6 and $24.5 \sigma$. 
Parameters and statistical significances of the $D_{J}$ resonances.

\begin{tabular}{c|c|c|c|c}
\hline \hline Resonance & $\begin{array}{c}\text { Final } \\
\text { state }\end{array}$ & $\begin{array}{c}\text { Mass } \\
{\left[\mathrm{MeV} / c^{2}\right]}\end{array}$ & $\begin{array}{c}\text { Width } \\
{\left[\mathrm{MeV} / c^{2}\right]}\end{array}$ & $\begin{array}{c}\text { Signif. } \\
(\sigma)\end{array}$ \\
\hline$D_{1}(2420)^{0}$ & $D^{*+} \pi^{-}$ & $2419.6 \pm 0.1 \pm 0.7$ & $35.2 \pm 0.4 \pm 0.9$ & \\
$D_{2}^{*}(2460)^{0}$ & $D^{*+} \pi^{-}$ & $2460.4 \pm 0.4 \pm 1.2$ & $43.2 \pm 1.2 \pm 3.0$ & \\
$D_{J}^{*}(2650)^{0}$ & $D^{*+} \pi^{-}$ & $2649.2 \pm 3.5 \pm 3.5$ & $140.2 \pm 17.1 \pm 18.6$ & 24.5 \\
$D_{J}^{*}(2760)^{0}$ & $D^{*+} \pi^{-}$ & $2761.1 \pm 5.1 \pm 6.5$ & $74.4 \pm 3.4 \pm 37.0$ & 10.2 \\
$D_{J}^{*}(2580)^{0}$ & $D^{*+} \pi^{-}$ & $2579.5 \pm 3.4 \pm 5.5$ & $177.5 \pm 17.8 \pm 46.0$ & 18.8 \\
$D_{J}^{*}(2740)^{0}$ & $D^{*+} \pi^{-}$ & $2737.0 \pm 3.5 \pm 11.2$ & $73.2 \pm 13.4 \pm 25.0$ & 7.2 \\
$D_{J}^{*}(3000)^{0}$ & $D^{*+} \pi^{-}$ & $2971.8 \pm 8.7$ & $188.1 \pm 44.8$ & 9.0 \\
\hline$D_{2}^{*}(2460)^{0}$ & $D^{+} \pi^{-}$ & $2460.4 \pm 0.1 \pm 0.1$ & $45.6 \pm 0.4 \pm 1.1$ & \\
$D_{J}^{*}(2760)^{0}$ & $D^{+} \pi^{-}$ & $2760.1 \pm 1.1 \pm 3.7$ & $74.4 \pm 3.4 \pm 19.1$ & 17.3 \\
$D_{J}^{*}(3000)^{0}$ & $D^{+} \pi^{-}$ & $3008.1 \pm 4.0$ & $110.5 \pm 11.5$ & 21.2 \\
\hline$D_{2}^{*}(2460)^{+}$ & $D^{0} \pi^{+}$ & $2463.1 \pm 0.2 \pm 0.6$ & $48.6 \pm 1.3 \pm 1.9$ & \\
$D_{J}^{*}(2760)^{+}$ & $D^{0} \pi^{+}$ & $2771.7 \pm 1.7 \pm 3.8$ & $66.7 \pm 6.6 \pm 10.5$ & 18.8 \\
$D_{J}^{*}(3000)^{+}$ & $D^{0} \pi^{+}$ & 3008.1 (fixed) & 110.5 (fixed) & 6.6
\end{tabular}

Among the processes studied, the three-body system $D^{*+} \pi^{-}$allowed for a spin analysis of the resonances produced and a separation of different spinparity components. The distributions of the helicity angle $\theta_{\mathrm{H}}$, between the momenta of $\pi^{-}$and the $\pi^{+}$from the $D^{*+}$ decay (as measured in the rest frame of the $D^{*+} \pi^{-}$system), have been fitted. As a result, natural parities were assigned to the resonances $D_{J}^{*}(2650)^{0}$ and $D_{J}^{*}(2760)^{0}$, while the states $D_{J}(2580)^{0}$ and $D_{J}(2740)^{0}$ were identified as unnatural parity ones. The structure observed close to $3000 \mathrm{MeV} / c^{2}$, denoted as $D_{J}^{(*)}(3000)$, is most probably a superposition of several states. The LHCb Collaboration has also performed a similar study of the $D_{s J}$ mesons decaying to $D^{+} K_{\mathrm{S}}^{0}$ and $D^{0} K^{+}$final states [31].

\subsection{Search for the doubly charmed baryon $\Xi_{c c}^{+}$}

The LHCb Collaboration has performed a search for the state $\Xi_{c c}^{+}$in the decay mode $\Xi_{c c}^{+} \rightarrow \Lambda_{c}^{+} K^{-} \pi^{+}$[32]. Predictions for its mass and lifetime span the ranges (3500-3700) $\mathrm{MeV} / c^{2}$ and (100-250) fs, respectively (see references in [32]). A signal for the $\Xi_{c c}^{+}$baryon in the abovementioned final state and with a mass of $(3519 \pm 2) \mathrm{MeV} / c^{2}$ was reported by the SELEX Collaboration, using a hyperon beam on a fixed target [33]. However, this observation was not confirmed in searches at three experiments: FOCUS [34], BaBar [35] and Belle [36]. The study at $\mathrm{LHCb}$ [32] also yielded a negative result. Upper limits on the $\Xi_{c c}^{+}$were obtained for a range of masses and lifetime hypotheses. 


\section{Summary}

In the recent years, the $\mathrm{LHCb}$ experiment has carried out an extensive programme of studies in the charm sector. It yielded, in particular, the first single-experiment observation of charm mixing. The searches for $\mathrm{CP}$ violation show no significant evidence in spite of a qualitative improvement in sensitivity to compare with previous studies. $\mathrm{LHCb}$ has also provided several important contributions to the spectroscopy of charmed hadrons. Most of these studies will be updated soon, with the addition of the data sample of $2 \mathrm{fb}^{-1}$ collected in 2012 .

This research was supported in part by PL-Grid Infrastructure.

\section{REFERENCES}

[1] W.N. Cottingham, D.A. Greenwood, An Introduction to the Standard Model of Particle Physics, Cambridge University Press, 1999.

[2] N. Cabibbo, Phys. Rev. Lett. 10, 531 (1963); M. Kobayashi, T. Maskawa, Prog. Theor. Phys. 49, 652 (1973).

[3] A.A. Alves, Jr. et al. [LHCb Collaboration], JINST 3, S08005 (2008).

[4] R. Aaij et al. [LHCb Collaboration], Nucl. Phys. B871, 1 (2013).

[5] R. Aaij et al. [LHCb Collaboration], Phys. Lett. B694, 209 (2010).

[6] A.A. Petrov [LHCb Collaboration], Int. J. Mod. Phys. A21, 5686 (2006).

[7] P. Spradlin [LHCb Collaboration], preprint LHCb-PROC-2013-076.

[8] B. Aubert et al. [BaBar Collaboration], Phys. Rev. Lett. 98, 211802 (2007).

[9] M. Staric et al. [Belle Collaboration], Phys. Rev. Lett. 98, 211803 (2007).

[10] T. Aaltonen et al. [CDF Collaboration], Phys. Rev. Lett. 100, 121802 (2008).

[11] Heavy Flavor Averaging Group, http://www.slac.stanford.edu/xorg/hfag/index.html

[12] R. Aaij et al. [LHCb Collaboration], Phys. Rev. Lett. 110, 101802 (2013).

[13] T. Aaltonen et al. [CDF Collaboration], arXiv:1309.4078 [hep-ex].

[14] B.R. Ko et al. [Belle Collaboration], arXiv:1401.3402 [hep-ex].

[15] R. Aaij et al. [LHCb Collaboration], Phys. Rev. Lett. 111, 251801 (2013).

[16] R. Aaij et al. [LHCb Collaboration], preprint LHCb-CONF-2013-003.

[17] R. Aaij et al. [LHCb Collaboration], Phys. Lett. B723, 33 (2013).

[18] M. Gersabeck et al., J. Phys. G 39, 045005 (2012).

[19] R. Aaij et al. [LHCb Collaboration], Phys. Rev. Lett. 112, 041801 (2014).

[20] R. Aaij et al. [LHCb Collaboration], J. High Energy Phys. 1306, 112 (2013).

[21] R. Aaij et al. [LHCb Collaboration], Phys. Lett. B726, 623 (2013).

[22] R. Aaij et al. [LHCb Collaboration], Phys. Lett. B728, 585 (2014). 
[23] S.-K. Choi et al. [Belle Collaboration], Phys. Rev. Lett. 91, 262001 (2003).

[24] B. Aubert et al. [BaBar Collaboration], Phys. Rev. D74, 071101 (2006).

[25] V. Bhardwaj et al. [Belle Collaboration], Phys. Rev. Lett. 107, 091803 (2011).

[26] N.A. Tornqvist, Phys. Lett. B590, 209 (2004).

[27] L. Maiani et al., Phys. Rev. D71, 014028 (2005).

[28] R. Aaij et al. [LHCb Collaboration], Eur. Phys. J. C72, 1972 (2012).

[29] S. Godfrey, N. Isgur, Phys. Rev. D32, 189 (1985).

[30] R. Aaij et al. [LHCb Collaboration], J. High Energy Phys. 1309, 145 (2013).

[31] R. Aaij et al. [LHCb Collaboration], J. High Energy Phys. 1210, 151 (2012).

[32] R. Aaij et al. [LHCb Collaboration], J. High Energy Phys. 1312, 090 (2013).

[33] M. Mattson et al. [SELEX Collaboration], Phys. Rev. Lett. 89, 112001 (2002).

[34] S. Ratti et al. [FOCUS Collaboration], Nucl. Phys. Proc. Suppl. 115, 33 (2003).

[35] B. Aubert et al. [BaBar Collaboration], Phys. Rev. D74, 011103 (2006).

[36] R. Chistov et al. [Belle Collaboration], Phys. Rev. Lett. 97, 162001 (2006). 